\title{
Geospatial analysis and decision support for health services planning in Uganda
}

\author{
Shuaib Lwasa \\ Urban Harvest, International Potato Center, Kampala, Uganda
}

\begin{abstract}
As the utilization of geospatial techniques continues to surge, spatial information has become an integral part of decision-making. In Uganda, the use of geospatial techniques in provision of health services planning has gained momentum after a comprehensive survey of health units and the development of a national health services geodatabase. Planning for the provision of health infrastructure services requires quality information to rationalize the location, and allocation, of services in relation to the population. Health service planners are always faced with a question of where to locate services in relation to need and how such distribution would be affected by resources to meet the requirements of the population. Because resources are scarce, prioritization is indispensable and thorough analysis becomes important in the planning process. This paper analyzes access to health facilities using the population gridding approach, coupled with location of health infrastructure facilities for decision support in health services planning.
\end{abstract}

Keywords: population gridding, location-based services, health planning, Uganda.

\section{Introduction}

The role of geospatial technologies in planning and management of location-based services is underscored by the several studies related to social services provision. The health infrastructure in Uganda (and elsewhere) depends on the physical structure and supporting equipment established for provision of health services. It usually involves facilities for different health service needs, equipment such as cold-chain facilities for storage and management tools for the distribution of health services to the population $(\mathrm{MoH}, 2002)$. Like in other developing countries of sub-Saharan Africa, health systems in Uganda are increasingly facing challenges in ensuring health care to its population (Leonard and Masatu, 2007; Mullan, 2007).

Challenges are posed by a number of factors,

Corresponding author:

Shuaib Lwasa

Urban Harvest, International Potato Center

Naguru Hill, Katarima Road, Plot 106

P.O. Box 22274

Kampala, Uganda

Tel. +256 41 4287571; Fax +25677 2461727

E-mail: s.lwasa@cgiar.org; lwasa_s@arts.mak.ac.ug including population growth, uneven population distribution in relation to the natural resource bases, low access by some areas due to limited transportation networks, availability of human resources and financial requirements for managing and provision of the health services. There is evidence that poor access to health care can be counter productive to growth and development and that rural communities are particularly vulnerable to the consequences of inaccessibility to health services (Leonard and Masatu, 2007). Health service provision is one of the many basic location-based social services that need to be provided in-tandem with the spatial distribution of the population. Planning for the provision of health infrastructure therefore requires quality information on location of services, capacity of facilities, population and catchment distance (the distance traveled by the furthest accessing patient to a health facility). Although a country's population requires proximity of health infrastructure and health care, some areas are served by distant facilities because of resource constraints. Prioritization of health service location therefore considers several factors, need and gaps being important among them (Katz et al., 2006). According to the national health 
policy (MoH, 2002), health infrastructures, supporting populations in rural as well as in urban areas, are to be established in various administrative units within $2 \mathrm{~km}$ reach. The Uganda Bureau of Statistics (UBOS, 2002) conducted a socio-economic survey on health service access and the result indicates that, within $5 \mathrm{~km}$ radius, the national average of health facility access is $73.2 \%$ with $69.6 \%$ for the rural population and $95.8 \%$ for the urban population. This was considered an improvement from the staggering proportion of the population living at the $10 \mathrm{~km}$ limit in 1991. Access to health services is close to the policy target of $2 \mathrm{~km}$ radius for urban areas but not for rural areas. This rural-urban quality divide can be explained by focusing on several issues, but it seems that the number of people served by a health facility and the distance to the nearest health facility are important indicators (Abel-Smith and Rawal, 1992; Shrestha, 2000; Leonard and Masatu, 2007).

This paper analyzes access to health facilities coupling population and location of health infrastructure facilities for decision support (Clancy and Cronin, 2005). The analysis is intended to enhance the understanding of location-based service analysis and provision of information for planning of health services.

\section{Decision-making in health services planning}

Decision-making is a process of solving a problem which is said to exist if "someone is in doubt as to which choice is best to remove his dissatisfaction with his present state" (Clancy and Cronin, 2005; WHO, 2006; O’Connor et al., 2007). Such a person, or in this context government, can identify three aspects related to the choices, namely:

(i) one or more outcomes that he/she desires;

(ii) two or more unequally efficient or effective courses of action; and

(iii) environment-containing factors that affect the outcomes (WHO, 2006).

In decision-making there is an ideal of behaving objectively and rationally in which optimal courses of action are found and relevant information for the decision are assumed to be readily available. But as Clancy and Cronin (2005) observed, information may not be readily available depending on the level and type of decision to be made. In addition, O'Connor et al. (2007) pointed out the importance of decision quality and supportive role of research coupled with decision-specific instruments for decision making. In practical terms decision-makers usually do not have all the relevant information when making decisions because of the time and cost constraints in gathering such information (Tunis et al., 2007). A decision-maker will stop gathering once some information is available on the basis of which a decision can be reached. But more information, either directly gathered or analyzed from existing data, would probably yield better decisions as pointed out in the context of health services planning (Eisenberg, 2002; WHO, 2006). Thus decisions tend to focus on procedures that lead to a solution that may not necessarily be optimal.

The path usually followed in decision-making is the procedural rationality in which the course of action involves searching for a satisfying rather than an optimal alternative (Katz et al., 2006; Yates et al., 2006; Health Services Research, 2007). Information search and its evaluation are very critical and, when spatial data is considered, as depicted in Figure 1, available techniques for evaluation provide a wide range of possibilities for manipulation to ensure better decisions based on existing information (ILRI and CBS, 2002). For policy analysis, part of the decision-making process is how spatial information can be captured and what methods would be used to evaluate such information.

Decision-makers usually operate within a tight time frame with inadequate resources and information (WHO, 2006; Yates et al., 2006). As worded by Mullan (2007), they are influenced by special-interests, bureaucratic imperatives, and political forces whose visions extend no further than the next election cycle. The current health services planning system in Uganda utilizes population data linked to administrative units with different spatial attributes 
and thus do not "spatiallize" population for appropriate allocation.

Having discussed the process and importance of decision-making, it is prudent to also highlight its application in health services planning. Like other location-based services, health services are critically tied to the space in which populations live. As pointed out by Shrestha (2000), it is a challenge to provide health services acceptable when the population is unevenly distributed. Although Gardner et al. (2007) do not explicitly highlight the importance of geospatial techniques, the focus on information technology indicates that all possible courses of action in health services planning, location inclusive, need to be explored. The geospatial analytical techniques are invaluable in analyzing and visualizing service gaps and needs and their introduction represent a crucial step towards allocation for service delivery and improvement of health care (Yates et al., 2006).

\section{Overall purpose and objectives}

Location-based services for health delivery present a research and policy challenge in dealing with the spatial distribution of, and the relation of such services to, other variables including the population.

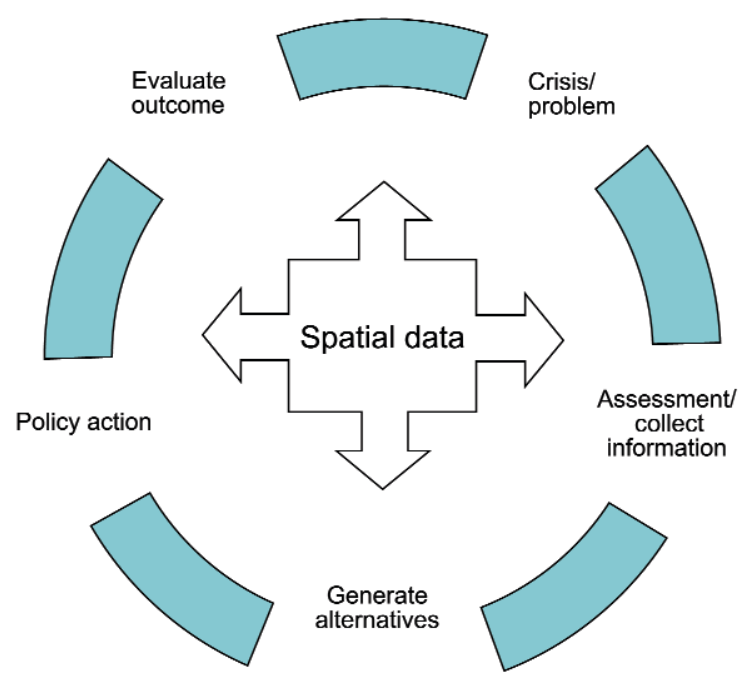

Fig. 1. The place of spatial data in the decision-making process.
Geospatial analytical techniques have been applied for the analysis of information much needed in decision-making. The data analysis can range from visualization, exploration through spatial statistics to spatial econometrics (Bivand, 1998; Jeong and Gluck, 2002; Davis, 2003). The underlying spatial relationships notwithstanding, exploration and visualization of data is very significant for decisionmaking because it clarifies where the need is or where hotspots and gaps exist. Such information provides a basis for evidence-based planning and management of services (Clancy and Cronin, 2005). Spatial observations upon which analysis can be undertaken include fields or surfaces, point patterns and lattice observations where attribute values relate to a grid, e.g. an administrative unit (Bivand, 1998).

The present study addresses the question how enhanced information regarding the relationships between services, capacity and catchment distance on one hand, and the population distribution on the other, can improve decision-making in the planning of health services. This question emerged from the fact that current services planning mechanisms are largely based on econometric models which could possibly be enhanced if coupled with spatial analytical techniques. In this way, the historical explanations of locating services could not only be explored but pointers for policy on what actions to be taken would also be provided (Walsh et al., 1993). The study employs triangulation of spatial techniques, including population gridding and spatial aggregation of health services using spatial statistics to generate information for improved decision-making.

The specific objectives were to spatially analyze health facility access by relating location and population, including:

(i) application of location-based analyzes of access to health facilities in Uganda;

(ii) generation of spatial information for visualization and support planning and delivery of health services; and

(iii) demonstration of the use of geospatial information and techniques in provision of information required for planning and health services delivery. 


\section{Materials and methods}

\section{Population data}

Population data utilized in the analysis were derived from the results of the Population and Housing Census of Uganda (UBOS, 2002). The data were captured as aggregate data for administrative units of parishes, the second-level administrative unit in Uganda, and detailed enough for the nationallevel type of analysis. The choice of parish-level population data was determined by the unavailability of readily usable spatial data layer for the lowest unit, i.e. the village. This population data is available in tabular form with linked data and parish labels. With the shapefile (NFA, 1996) of the national administrative layer, the data was entered into the geodatabase for further processing. Processing of the data included adjustment of the population with the annual growth rate to compute estimates for the year 2006 (UBOS, 2005). To that end, the population estimates used in the analysis were adjusted using the annual growth rate of $3.2 \%$. Although this presents a problem of unifying the growth rates for all administrative units, the resultant total estimate was considered accurate enough for the purpose of this study.

\section{Administrative boundary data and bealth facilities GPS point data}

Spatially-explicit data were acquired from the updated national administrative geodatabase (NFA, 1996). Although some boundary changes were noted, especially at the sub-county and district levels, the parish-level boundaries had not significantly changed. Geospatial analytical techniques were utilized to process the data for the analysis. A grid tool providing the opportunity to determine spatial resolution enabled generation of a regular square spatial layer that was geo-referenced and linked to the administrative boundary of Uganda. The processing of the spatial distribution of the population involved several steps, for example, excluding water bodies from the administrative layer, to enable a more accurate estimate of the population density. The resultant map layer was overlayed with the grid layer and population estimates calculated for each area unit and later aggregated to each grid. Because a grid is a square cell, the process produced area units which were disected by the administrative boundary layers during overlay to enable calculation of population of each unit area based on the density and area of the disaggregated areal units of the grids (Diechmann and Balk, 2001).

In this study a $5 \mathrm{~km}$ spatial resolution, as described in de By (2004), was utilized and the result of this process was a spatial layer with a population estimate for each grid (Fig. 2). This technique is more robust than administrative-based summarizes which are not spatially-populated and where the population densities include the water bodies. In addition, the health infrastructure database was acquired from the geodatabase created by global positioning system (GPS) data which had been captured from 2000 to 2003 and continuously updated. Additional data available from the health facilities geodatabase include health facility grades and type of ownership, catchment distance, and bed capacity which was also collected during the GPS point survey.

Geostatistical analysis (Anselin, 2004) and ArcGIS were utilized to spatially explore the data, visualize and statistically analyze relationships between the key variables of population, number of health facilities, average catchment distance and bed capacity. The data were explored in terms of location randomness within the grids. The study utilized the regression model and Moran's I statistic for relationships between variables generating significance maps which were visualized to enhance understanding of access to health by distance and the spatial distribution of the facilities.

\section{Results and discussion}

\section{Exploration of population data}

To establish where people are generally concentrated, the population was "spatiallized" using a 


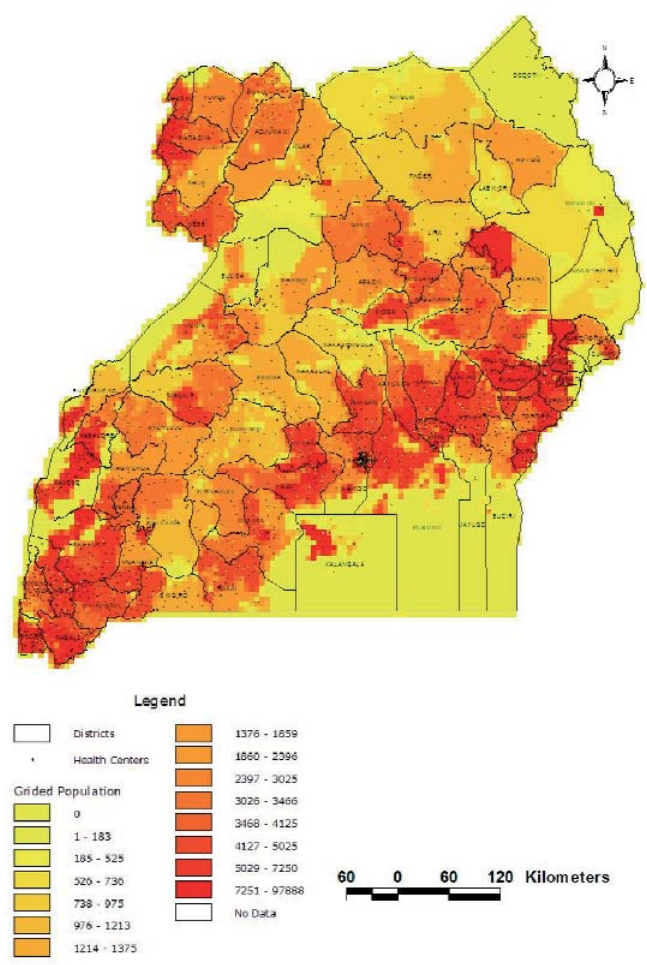

Fig. 2. Grided population of Uganda at $5 \times 5 \mathrm{~km}$ resolution.

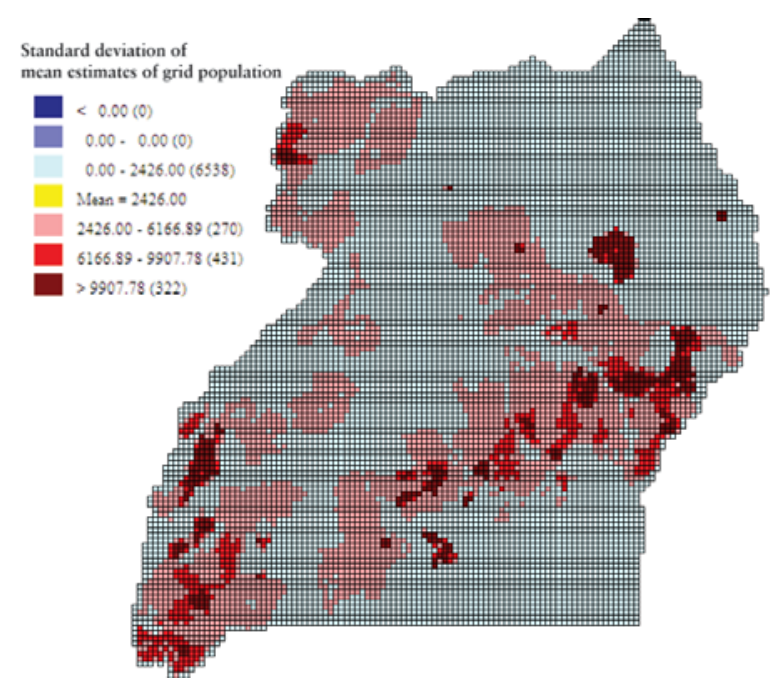

Fig. 3. Gridded population of Uganda showing areas of concentration. gridding approach (Diechmann and Balk, 2001; Davis, 2003) regarded as fairly accurate. The gridded dataset is utilized to visualize the spatial distribution of population in Uganda before relating it to provision of services. Decision-making requires robust data on location of services and population which provides the demand of services. Exploration of population distribution in Uganda indicates concentration of population in areas around the major water bodies of Lake Victoria and Lake Kioga, and the mountainous areas although there are a few outliers, especially in the areas of conflict in the North and North-East. As shown in Figure 3, the exploration also revealed smaller concentrations in areas with populations less than the mean of the grids. The implication of this analysis is that location of services needs to be sensitive and responsive to population distribution. A further analysis of the data indicates that 251 grids have populations in the upper outlier implying high concentration of population in relatively small areas which are the areas of current conflict. This distribution has implications for services location and planning and, later in the paper, is utilized to analyze the relationship between population and services to generate information useful for decision-making.

\section{Health infrastructure development in Uganda}

The Ministry of Health $(\mathrm{MoH})$ in Uganda has a programme for health infrastructure construction. It equips the health centers (HC) across the country which are designated as HC II, HC III, HC IV and hospital ${ }^{1}$ according to the grade of sophistication. This programme is a response to the need for improving access to basic need of health recognizing the inadequacy of health care facilities (UNDP, 2005). In addition to the quality of staff associated

\footnotetext{
1 HC II, health center II serves an administrative unit which is a parish; HC III, health center III serves an administrative unit which is a sub-county; HC IV, health center IV, also termed health sub-district headquarter, serves constituency; hospital, serves as district or can be a regional referral health unit.
} 
with each grade, all health facilities in Uganda are graded based on the health services offered and the administrative or political unit serviced. The HC II are managed by nursing officers and the services include treatment of non-complicated ailments that require clinical services, bed rests for some patients and they act as points for near-neighbourhood advise on health care (Shrestha, 2000). The HC III services a sub-county and are managed by a clinical officer and their activities include maternal health care, a labour ward, non-complicated surgery services and general treatment. An HC IV is managed by a qualified doctor, has a labour ward, surgery theatre, offers general health-care besides supervising all the lower level health centers in a constituency, and provides outreach programmes and guidance. The hospital represents the highest level available. It offers all health care services, can have several qualified doctors as well as lower level health workers, manages outreach programmes, and some serve as regional referrals for complicated ailments. This health services tier is linked to the administrative units and the higher administrative levels in which management responsibility is also shared between local governments such as districts, sub-counties or parishes. The highest authority in the medical area rests with $\mathrm{MoH}$ and the Government. An implicit assumption in health services provision is that the less the distance to the health facility, the more access to health services.

According to UBOS (2005) the access to health facilities and its services has improved from $49 \%$ coverage in 1992 to $69.9 \%$ in 2005 for the population living within $5 \mathrm{~km}$ of a health service unit. However, this is based on social surveys conducted during the census which may not accurately provide for the distance factor. The challenge is that the distance measurements are interpreted differently by the many rural communities in Uganda and, consequently, self-reported distance data may not always be correctly represented in the surveys. Obviously, the distance influence the level of access to the health services since the transportation of sick people, and the time it takes, have a bearing on the response and action taken. Rural communities are particularly affected because there are still marked variations in access to the health facilities both within and between districts (Harrison and Verhoef, 2002; Barber et al., 2007; Leonard and Masatu, 2007). Beyond physical access, many of these health facilities do not provide the full range of essential primary health care services. Country wide, there is a total of 2314 health facilities of all grades and their distribution by district is shown in Figure 2 . The distribution of health centers by grade is analyzed in Table 1 . The HC II distribution appears satisfactory but that of HC III, HC IV and hospitals still requires improvement, especially in relation to the population served and the services offered.

Analysis of access to bealth facilities based on distance

Physical access to health facilities is critical in health services planning since the distance to a health facility is significantly associated with morbidity. Although the literature indicates improvement in health service access (NEMA, 2000/01; UBOS, 2005 ) and there is continued investment in healthrelated infrastructures, especially in rural areas, there are still questions regarding access to health services in relation to population distribution. The level of access to services has been analyzed previously on the basis of a survey conducted country-wide (UBOS, 2005). In my opinion, however, it does not adequately portray the access levels. Given the shortcomings of self-reported data and the many interpretations of distance by the various ethnic groups in the country, there is a need to verify the reported sta-

Table 1. Descriptive statistics of health facilities in Uganda, stratified by grade.

\begin{tabular}{lrrc}
\hline $\begin{array}{l}\text { Health } \\
\text { center }\end{array}$ & Total & Mean & $\begin{array}{c}\text { Standard } \\
\text { deviation }\end{array}$ \\
\hline HC II & 1268 & 23 & 20 \\
HC III & 778 & 14 & 13 \\
HC IV & 159 & 3 & 2 \\
Hospital & 109 & 2 & 2 \\
Total & 2314 & & \\
\hline
\end{tabular}


tistics with more robust, spatial analytical techniques to identify gaps and needs in access to health facilities. The analysis of coverage, as presented in this paper, has been enhanced with spatially-explicit access information which integrates gridded population with location of health facilities.

Using the grid approach, and applying the spatial analytical technique of proximity analysis with a tight distance algorithm, the analysis revealed that an estimated 6.6 million people $(27.3 \%$ of the population of Uganda) live within the $5 \mathrm{~km}$ radius of a health facility. When touching areas were included, the proportion increased to $36.3 \%$. A further analysis of access revealed that there are differences in access determined by the location of the health facility within the grid. A centrality assessment returned $60.4 \%$ proportion of health facilities at more than $4.2 \mathrm{~km}$ from the centroid of the grid which denotes a random location of health facilities within each grid. It should be noted that the importance of method and algorithm for generating the grid need not to be underestimated for the centrality results. Figure 4 shows the distribution of people based on grids with a $5 \mathrm{~km}$ radius and the concentrations in the center-east and south-west, while Figure 5 shows the distribution of government and non-government health facilities. Few grids are exclusively served by facilities run by non-governmental organizations (NGOs), but if the need is to analyze information on the nature of the NGOs, it is important in determining the life-span of the facility. The implication is that ownership and operational status is critical in the analysis. Some NGO-based health facilities tend to have a shorter life-span than government-owned facilities and this is especially true for the lower-level health centers. In addition, when it comes to functionality, some NGO-operated health centers suffer from intermittent operation due to drug unavailability and inadequacy of personnel.

As shown in Figures 6 and 7, it is obvious that from the grids in yellow, that part of the central region around Lake Victoria, as well as scattered areas in the eastern and south-western parts of the country, have better access to health facilities than is

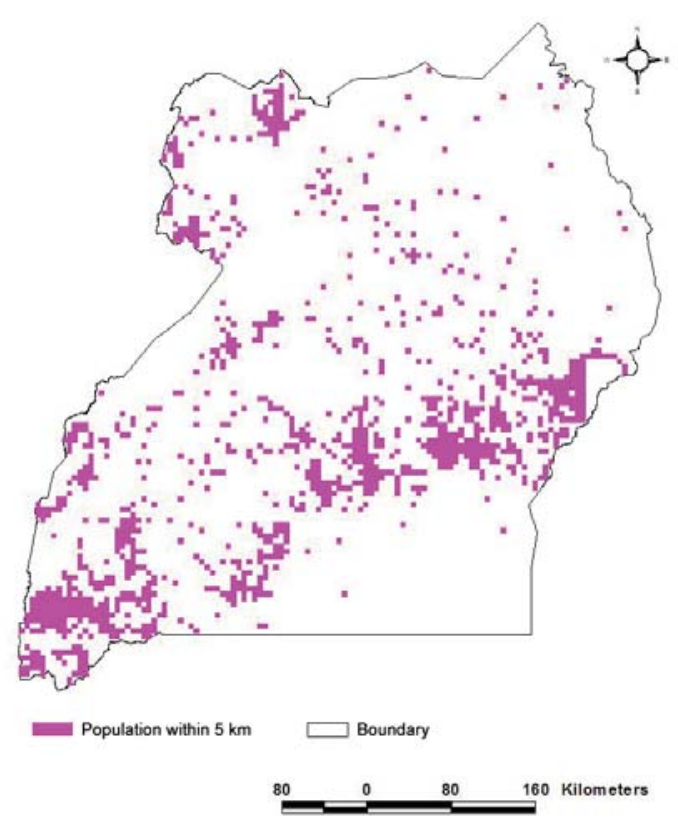

Fig. 4. Populated areas within $5 \mathrm{~km}$ access to health facilities in Uganda.

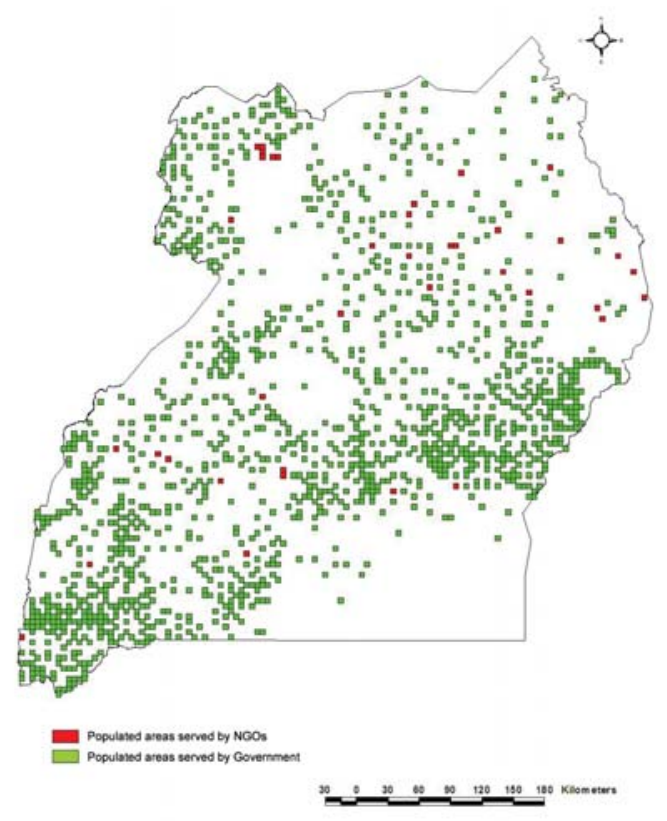

Fig. 5. Populated areas served by government facilities and NGOs in Uganda. 


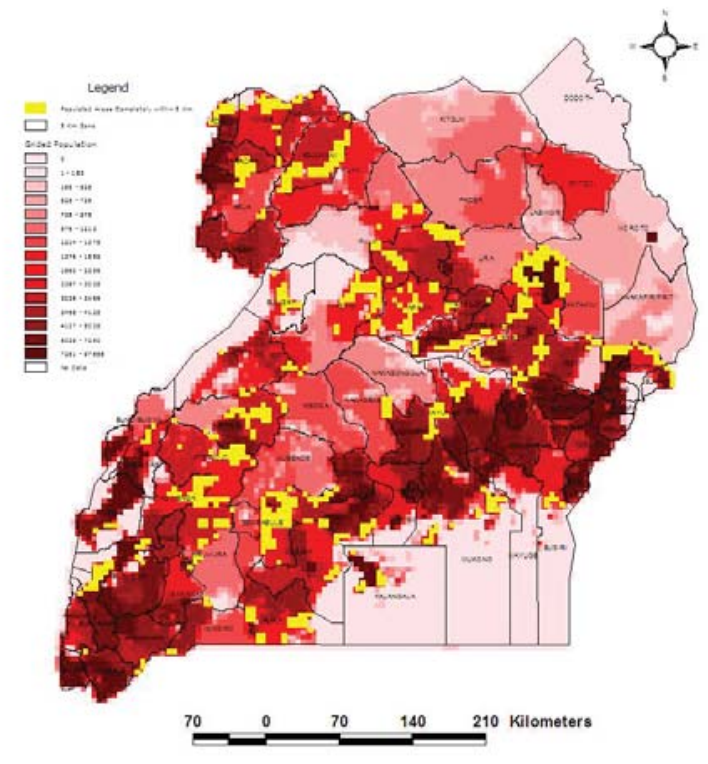

Fig. 6. Populated areas completely within $5 \mathrm{~km}$ radius in Uganda.

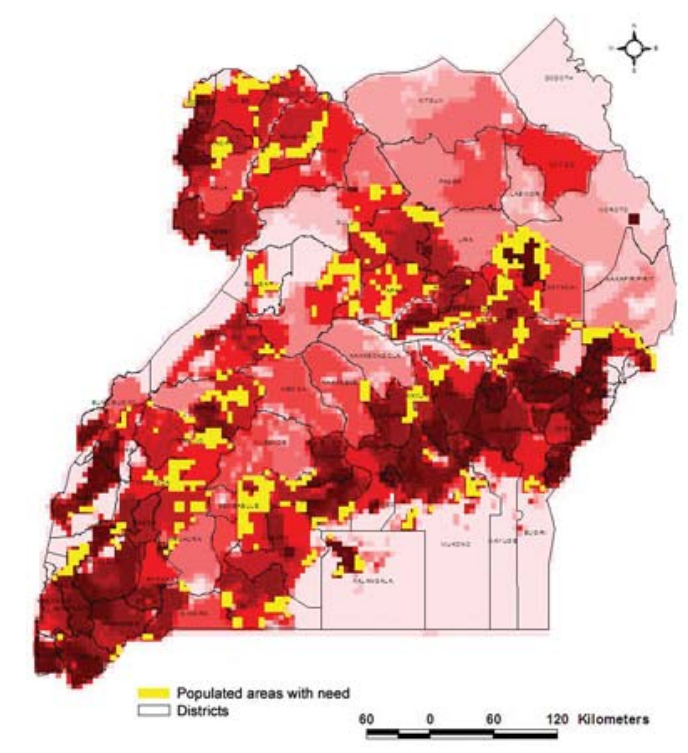

Fig. 7. Populated areas with greatest need, population $>1500$ and distance of $>10 \mathrm{~km}$ in Uganda. the case in the central, northern and north-western regions. The clustering of health facilities around Lake Victoria is clearly due to the higher population numbers and the commercial connectivity of the region, while in the north-west, the presence of refugees and international organizations such as the World Food Program, the UN Human Center for Refugees explains the higher concentration of health facilities in some of the districts.

\section{Relating population to the location of health services}

As noted earlier, the research question that this paper attempts to address is whether the spatial distribution of population and the location of services relate with key variables including bed capacity and catchment distance in trying to generate information vital for decision-making regarding the planning of health services. Access to health facilities for the population was also recognized in the national population policy as a key ingredient of sustainable development (GoU, 1995). Utilizing spatial analysis techniques, the key variables of population and number of facilities by grid were plotted to establish any relationships. The result (Fig. 8) indicated that an increase in population is correlated with an increase in number of facilities. A further analysis of the relationship using Moran's I statistic (Fig. 9) indicates a weak but significantly positive relationship between population and the number of facilities $\left(\mathrm{R}^{2}=0.193\right)$. The interpretation of this statistic is that population, in as much it is an important variable in planning for health facilities, is not statistically significant in the historical location of facilities. On the other hand, it reveals the need for consideration of population in locating health services.

The local index spatial autocorrelation, as shown in Figure 10, also indicates the spatial significance of the relationship between population and the number of facilities. The computed results show this, with a high statistical significance $(\mathrm{p}<0.001)$, in areas of high population and high number of health facilities. Two important scenarios can be discerned 


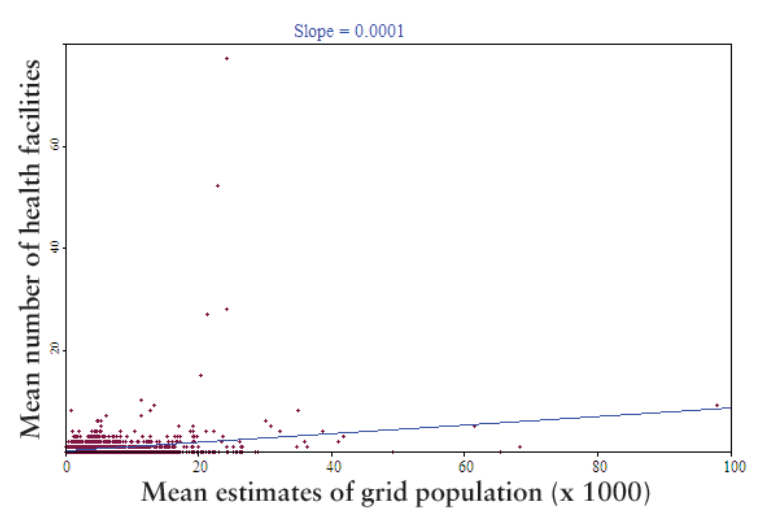

Fig. 8. Scattergram of grid population and number of health facilities in Uganda.

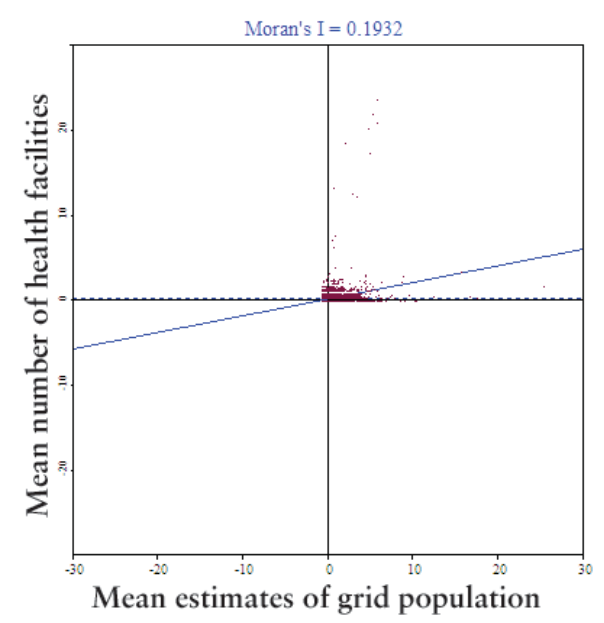

Fig. 9. Bivariate Moran's I of grid population and number of health facilities in Uganda.

from the significance map: firstly, areas with high population and high number of health facilities, which according to the map are categorized as highhigh, and secondly, areas with high population but with a low number of health facilities. Whereas the former might depict 'appropriate allocation', the later reveals under-allocation of health facilities. This represents a gap that needs to be filled by health services and we come to this conclusion based on fairly accurate information. In terms of decision-making, visualizing the maps such as Figure 9, should communicate where improvement is required to decision-makers more readily than the provision of such information in terms of tables and graphs.

A spatial regression statistical model using least squares also reveals a positive but weak causaleffect relationship between population and the number of facilities. The regression model was applied to estimate the prediction of the number of facilities by population. The results indicate that $\mathrm{R}^{2}=0.068$ at a significance level of $\mathrm{p}<0.001$ with a low spatial dependence. The importance of this statistic emphasizes the need for consideration of the population distribution to improve access when planning localization of health services. The facilities variable was also tested against the average catchment distance using recorded data regarding the geographical origin of the patients. To smooth the data and remove errors of estimate the range distance was computed for each health facility. The data were then linked to the health facilities and spatially joined to be identified with the grid and its estimated population. The results indicate a weak prediction $(\mathrm{p}<0.001)$ of the average catchment distance $\left(\mathrm{R}^{2}<0.001\right)$ by the number of health facilities per grid. The assumption when testing this relationship was that the average catchment distance would increase with the number of health facilities since the services would be available to the population, not only within the administrative unit serviced but from neighbouring units as well. The implication of this analysis is that even if health facilities are located in a given administrative area, they will not necessarily guarantee access to the services to neighbouring administrative units. This fact takes into consideration the need to factor in issues such as remoteness, physical constraints and social issues of preference.

In addition, the catchment distance was analyzed in relation to bed capacity and the assumption was that the average catchment distance would predict the bed capacity. Although results indicated 


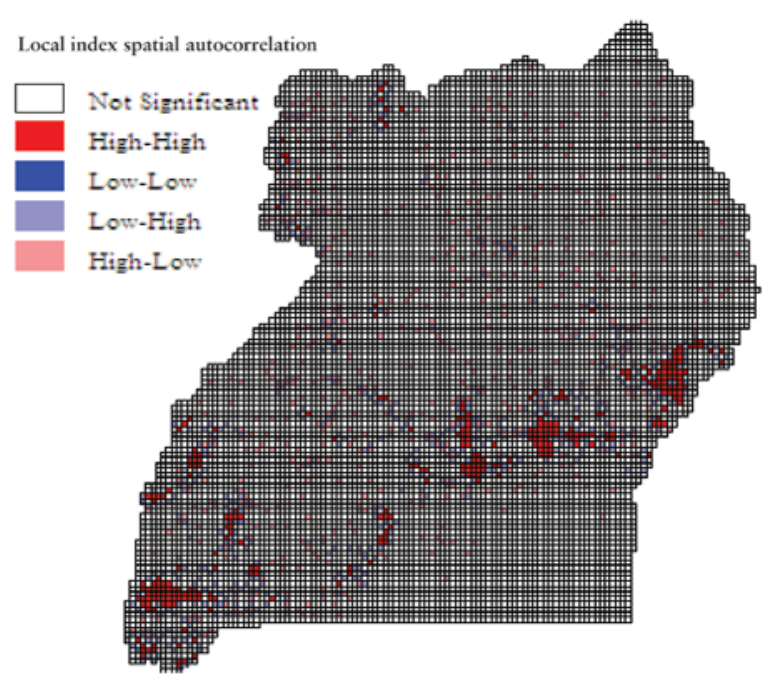

Fig. 10. Local index spatial autocorrelation of grid population and number of health facilities in Uganda.

$\mathrm{R}^{2}=0.093$ at the significance level of $\mathrm{p}=0.065$, there is a high degree of dependence between these two variables. These statistical results do not only satisfy research needs but also underline their potential in health services planning because of the revelation of underlying relationships. Bed capacity analysis is crucial in treating complicated ailments such as malaria and other diseases which require rest or admission. Although admissions data would be most appropriate in this analysis, average catchment distance gives an indication of service reach in space and with that the population distribution can be utilized in planning for bed capacity. The following section attests to the implications of the spatial analysis in planning for health services in Uganda.

\section{Implications for health services planning}

As indicated in the spatial data exploration as well as statistical analyses, and coupled with the importance of public decision-making for improvement of health services, this section of the paper discusses the results in the context of services planning. Population distribution is an important factor in planning the location of health services. While the need for health facilities is obvious in areas with population concentrations and where the current access distance is higher than the national target of $5 \mathrm{~km}$, it is critical where the distance exceed $10 \mathrm{~km}$. In Figure 7, the populated grids where the need is high show a dispersed population with facilities more than $10 \mathrm{~km}$ away and a grid population of $>1500$ persons. The number of people living in such areas is 2.8 million, i.e. $11.5 \%$ of the national population as shown on Figure 6 (yellow cells). In the conflict districts of eastern Uganda, the presence of internally displaced people's camps creates a great need for health services, while in the central-northern part of the country, the need is also clearly displayed. In the context of prioritization, health facilities planning must consider where the areas of the greatest need are located, something which can be achieved with relative ease by the application of geospatial techniques. Information provided by these techniques holds promise to enhance decision-making for the health sector.

With regard to population-service in general, the statistical results show that the location of health facilities are not correlated with the spatial distribution of population. The implication is that better distance analyses are necessary and "spatializing" of the population can greatly improve health services planning by basing the construction of facilities on need and access. The statistical significance maps show where the gaps exist, i.e. where there are grid squares of high population but with a low number of health facilities. Further underscoring the population factor in location of health facilities, the maps also visualize areas where there are a high number of health facilities in spite of a low population. This is understandable because allocation is based on population aggregation by administrative units but can be improved with spatial distribution of population. The situation and the key findings can be summarized as follows: 
(i) health facilities are not located based on the spatial distribution of population;

(ii) health services are accessible based on distance as recognized by the planning authority but the $5 \mathrm{~km}$ distance threshold has a further implication that has to do with the time it takes to reach the facility; and

(iii) the gridding approach, despite weaknesses related to sourcing boundary data and aggregation of population data, provides a mechanism for adequately estimating the need for health services through visualization of the population distribution.

\section{Conclusions}

Geospatial analysis techniques and information can aid planning, decision-making and delivery of health services. By combining population and location of facilities in an innovative way using grids, locationbased services can be analyzed for gaps and needs. The historical location of health services in Uganda appears to have been based on administrative rather than population distribution. This is derived from the statistical analysis results which indicate positive but weak prediction of facilities by population. It is also recognized that there are several means of analyzing health services access and distance is just one of them. Equally, there are shortcomings of the gridding approach especially in the estimation of population based on density. Despite these weaknesses, the geospatial approach can provide valuable information for health services planning.

\section{Acknowledgement}

The author wishes to thank the Ministry of Health in Uganda for support in preparing this paper including access to the geodatabase. Thanks also go to the National Forestry Authority, Uganda for the national administrative dataset and the Uganda Bureau of Statistics GIS unit for the updated administrative dataset. The health facilities dataset was compiled with funding from the Government of Uganda through the Ministry of Health.

\section{References}

Abel-Smith B, Rawal P, 1994. Employer's willingness to pay: the case for compulsory health insurance in Tanzania. Health Policy Plan 9, 409-418.

Anselin L, 2004. GeoDaTM 0.9.5-i Release Notes. Center for Spatially Integrated Social Science.

Barber SL, Gertler PJ, Harimurti P, 2007. The contribution of human resources for health to the quality of care in Indonesia. Health Aff (Millwood) 26, 367-379.

Bivand R, 1998. A review of spatial statistical techniques for location studies. Department of Geography, Norwegian School of Economics and Business Administration, 25 pp.

Clancy CM, Cronin K, 2005. Evidence-based decision making: global evidence, local decisions. Health Aff (Millwood) 24, 151-162.

Davis B, 2003. Choosing a method for poverty mapping. Agriculture and Economic Development Analysis Division, FAO (Food and Agriculture Organization of the United Nations), Rome, Italy.

de By RA (Editor), 2004. Principles of geographic information systems: an introductory textbook. ITC Educational Textbook Series 1, 230 pp.

Diechmann U, Balk D, 2001. Transforming population data for interdisciplinary usages: from census to grid. New York. Eisenberg JM, 2002. Globalize the evidence, localize the decision: evidence-based medicine and international diversity. Health Aff (Millwood) 21, 166-168.

Gardner CA, Acharya T, Yach D, 2007. Technological and social innovation: a unifying new paradigm for global health. Health Aff (Millwood) 26, 1052-1061.

GoU (Government of Uganda), 1995. National population policy for sustainable development. Kampala, Uganda.

Harrison A, Verhoef M, 2002. Understanding coordination of care from the consumer's perspective in a regional health system. Health Serv Res 37, 1031-1054.

Health Service Research, 2007. The performance of performance measurement. Health Serv Res 42, 1797-1801.

ILRI and CBS, 2002. Mapping poverty in Kenya and Uganda. International Livestock Research Institute, Nairobi, Kenya.

Jeong W, Gluck M, 2002. Multimodal bivariate thematic maps with auditory and haptic display. Proceedings of the 2002 International Conference on Auditory Display, Kyoto, 
Japan, July 2-5, 2002.

Katz A, Staiti AB, McKenzie KL, 2006. Preparing for the unknown, responding to the known: communities and public health preparedness. Health Aff (Millwood) 25, 946-957.

Leonard KL, Masatu MC, 2007. Variations in the quality of care accessible to rural communities in Tanzania. Health Aff (Millwood) 26, 380-392.

$\mathrm{MoH}, 2002$. National health policy. Kampala. Ministry of Health, Uganda.

Mullan F, 2007. Health, equity, and political economy: a conversation with Paul Farmer. Health Aff (Millwood) 26, 1062-1068.

NEMA (National Environmental Management Authority), 2000/01. State of the environment report for Uganda. Kampala, Uganda.

NFA (National Forestry Authority), 1996. Administrative areas of Uganda. Kampala, Uganda.

O’Connor AM, Wennberg JE, Legare F, Llewellyn-Thomas HA, Moulton BW, Sepucha KR, Sodano AG, King JS, 2007. Toward the 'tipping point': decision aids and informed patient choice. Health Aff (Millwood) 26, 716-725.

Shrestha LB, 2000. Population aging in developing countries. Health Aff (Millwood) 19, 204-212.

Tunis SR, Carino TV, Williams RD 2nd, Bach PB, 2007. Federal initiatives to support rapid learning about new technologies. Health Aff (Millwood) 26, 140-149.

UBOS, 2002. Uganda population and housing census. Kampala, Uganda, Bureau of Statistics.

UBOS, 2005. Statistical abstract. Kampala, Uganda, Bureau of Statistics.

UNDP, 2005. Human Development Report, 2005. International Cooperation at a Crossroads Aid, Trade and Security in an Unequal World, pp. 260.

Walsh DC, Rudd RE, Moeykens BA, Moloney TW, 1993. Social marketing for public health. Health Aff (Millwood) 12, 104-119.

WHO, 2006. Health service planning and policy making: a toolkit for nurses and midwives. Western Pacific Region, 48.

Yates J, Cooper R, Holland J, 2006. Social protection and health: experiences in Uganda. Dev Policy Rev 24, 339-356. 